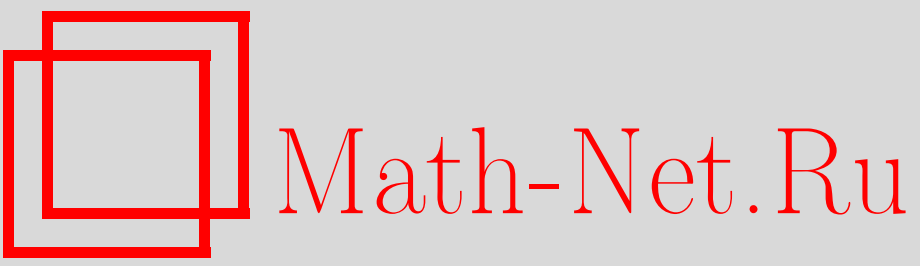

В. А. Дремов, Об одном p-адическом множестве Жюлиа, УМН, 2003, том 58, выпуск 6, 151-152

DOI: https://doi.org/10.4213/rm682

Использование Общероссийского математического портала Math-Net.Ru подразумевает, что вы прочитали и согласны с пользовательским соглашением

http://www.mathnet.ru/rus/agreement

Параметры загрузки:

IP: 3.82 .47 .9

26 апреля 2023 г., $14: 17: 34$ 


\section{ОБ ОДНОМ $p$-АДИЧЕСКОМ МНОЖЕСТВЕ ЖЮЛИА}

\section{В.А. ДРемов}

В последнее время интенсивно исследуются неархимедовы динамические системы; см., например, [1]. В данной работе исследуется класс динамических систем вида $x \mapsto x^{2}-\frac{a^{2}}{p^{2}}$ на множестве $p$-адических чисел. Рассматривается структура множества Жюлиа, определенного для этих систем по аналогии с классическим случаем, и описьвается действие на нем нашего отображения, которое сводится к символической динамике. При этом доказьвается, что все периодические точки лежат в основном поле.

1. ОПРЕДЕЛЕНИЯ.

$p$-адические числа. Зафиксируем нечетное простое $p$.

$\mathbb{Q}_{p}$ - поле $p$-адических чисел, $\mathbb{Z}_{p}$ - кольцо $p$-адических чисел. См. [2].

Если $x \in \mathbb{Q}_{p}$, то $v(x)=k$, где $x=p^{k} r, k \in \mathbb{Z}, r \in \mathbb{Z}_{p} \backslash p \mathbb{Z}_{p}$.

$a \equiv b\left(\bmod p^{k}\right)$ означает, что $a-b \in p^{k} \mathbb{Z}_{p}$.

Рассматривается $\mathbb{P}^{1}\left(\mathbb{Q}_{p}\right)=\mathbb{Q}_{p} \sqcup\{\infty\}$ с соответсвующей топологией.

Специальные обозначения.

$T: \mathbb{P}^{1}\left(\mathbb{Q}_{p}\right) \rightarrow \mathbb{P}^{1}\left(\mathbb{Q}_{p}\right)$ есть квадратичное отображение $x \mapsto x^{2}-\frac{a^{2}}{p^{2}}$, где $v(a)=0$.

$x_{i}:=T^{i}\left(x_{0}\right)$ для $i \geqslant 1$.

$J:=$ множество Жюлиа - минимальное инвариантное относительно как $T$, так и $T^{-1}$ замкнутое множество, состоящее не менее чем из двух точек. (См. [3] по поводу равносильности с другими определениями.)

$K:=\left\{x \mid x_{n} \nrightarrow \infty\right.$ при $\left.n \rightarrow \infty\right\}$ (отображение $T$ выбрано так, что это множество невелико и совпадает с множеством Жюлиа).

2. Teopema. a) $J=K$.

б) $J$ является замыканием множества конечных периодических точек отображения $T$.

3. Лемма 3.1. $\forall x_{0}\left(v\left(x_{0}\right) \neq-1 \Rightarrow x_{n} \rightarrow \infty\right.$ npu $\left.n \rightarrow \infty\right)$.

СлеДСтвиЕ 3.2. $\forall x_{0}\left(x_{n} \nrightarrow \infty \Rightarrow \forall k \in \mathbb{N}_{0}\left(x_{k} \pm \frac{a}{p} \in \mathbb{Z}_{p}\right)\right)$.

Для доказательства используем тот факт, что $v\left(x_{k}\right)=v\left(x_{k+1}\right)=-1$.

ОПРЕДЕЛЕНИЕ-ОБОЗНАЧЕНИЕ 3.3 . Используя результат следствия 3.2 , получаем

$\exists ! \phi: K \rightarrow\{-1,1\}^{\mathbb{N}}$ такое, что $x_{n}-\frac{a}{p}\left(\phi(x)_{n}\right) \in \mathbb{Z}_{p}$.

УТВЕРЖДЕНИЕ 3.4. Отображение $\phi: K \rightarrow\{-1,1\}^{\mathbb{N}}$ вляется гомеоморфизмом. (Соответствующее пространство $\Sigma_{2}^{+}$вместе с отображением левого сдвига на нем определяется в [4]; см. также утверждение 3.5$)$.

ДокАЗАтЕльство. Рассматриваем следующую систему

$$
\left\{x_{0}-a_{0} \in \mathbb{Z}_{p} \& x_{1}-a_{1} \in \mathbb{Z}_{p} \& \ldots \& x_{s}-a_{s} \in \mathbb{Z}_{p}\right\} .
$$

Здесь $a_{0}, a_{1}, \ldots, a_{s} \in\left\{-\frac{a}{p}, \frac{a}{p}\right\}$.

Индукцией по $k$ доказьвается, что все решения этой системы с $s=k$ образуют множество вида $y(k)+p^{k} \mathbb{Z}_{p}$, где $y(k) \in p^{-1} \mathbb{Z}_{p}$.

Теперь заметим, что уравнение $\phi\left(x_{0}\right)=\left(\varepsilon_{0}, \varepsilon_{1}, \ldots\right)$ равносильно тому, что система вида $(1)$ вьполняется для всех $s$ при $a_{n}=\frac{a}{p} \varepsilon_{n}$. Значит, предел $\lim _{k \rightarrow \infty} y(k)$ не только существует, но и является единственным решением уравнения $\phi\left(x_{0}\right)=\left(\varepsilon_{0}, \varepsilon_{1}, \ldots\right)$. Отсюда мы получаем биективность. Непрерьвность $\phi$ и $\phi^{-1}$ тоже следует из утверждения, доказанного по индукции. 
УТВеРЖДЕнИЕ 3.5. Отображение $\phi: K \rightarrow\{-1,1\}^{\mathbb{N}}$ удовлетворяет соотношению $\phi T(x)=L \phi(x)$ для $x \in K$, где $L$ - отображение левого сдвига, $L:\left(\varepsilon_{0}, \varepsilon_{1}, \ldots, \varepsilon_{k}, \ldots\right) \mapsto$ $\left(\varepsilon_{1}, \varepsilon_{2}, \ldots, \varepsilon_{k+1}, \ldots\right)$.

Утверждение следует из биективности $\phi$ (утверждение 3.4) с учетом определения 3.3 .

УТВЕРЖДЕНИЕ 3.6. Все конечные периодические точки отображения ф лежат в $K$.

Для доказательства пользуемся утверждением 3.5 и тем, что количество конечных неподвижных точе к для $T^{n}$ не более $2^{n}$.

УТВеРЖДЕНИЕ 3.7. К совпадает с замыканием мно сества конечных периодических точек отображсения $T$.

ДокАЗАтЕЛЬСтво. Заметим, что $K$ - замкнуто, как пересечение замкнутых множеств вида $x+p^{k} \mathbb{Z}_{p}$ (см. доказательство утверждения 3.4$)$. А всюду плотность в $K$ множества периодических точек отображения $T$ следует из всюду плотности множества периодических последовательностей во множестве $\{-1,1\}^{\mathbb{N}}$ всех последовательностей.

Вопрос о совпадении множества Жюлиа и замькания множества отталкивающих точек отображения рассматривался в [3]. Там сформулирована гипотеза о том, что для любого рационального отображения эти множества равны. Рассмотрены также примеры, свидетельствующие в полшзу этой гипотезы.

ДокАЗАТЕЛЬСТво тЕОРЕмЫ. Сначала докажем утверждение а).

$K$ - инвариантно относительно $T$ и $T^{-1}$. Замкнутость $K$ следует из утверждения 3.7. Чтобы доказать минималность, достаточно рассматривать ограничение $T$ на $K$. Воспользуемся утверждением 3.5. Получим, что мы можем рассматривать только замкнутые инвариантные подмножества $Y$ в $\{-1,1\}^{\mathbb{N}}$ относительно действия $L$. Тогда, очевидно, $\forall y \in Y \forall k \in \mathbb{N}$, $\forall\left(a_{0}, a_{1}, \ldots, a_{k}\right) \in\{-1,1\}^{k+1}\left(Y \ni\left(a_{0}, a_{1}, \ldots, a_{k}, y_{0}, y_{1}, y_{2}, \ldots\right)\right)$. И тогда из замкнутости $Y$ следует, что $Y=\{-1,1\}^{\mathbb{N}}$.

Теперь утверждение б) следует из утверждения 3.7.

\section{СПИСОК ЛИТЕРАТУРЫ}

[1] A. Yu. Khrennikov. Non-Archimedean Analysis: Quantum Paradoxes, Dynamical Systems and Biological Models. Dordrecht: Kluwer, 1997. [2] Н. Коблиц. $p$-адические числа, $p$-адический анализ и дзета-функции. М.: Мир, 1982. [3] Liang-Chung Hsia // J. London Math. Soc. (2). 2000. V. 62. № 3. Р. 685-700. [4] Р. Боуэн. Методы символической динамики. М.: Мир, 1979 .

Принято редколлегией 23.09 .2003 